\title{
Wind Structure in Winter Storms
}

\section{R. H. SHERLOGK and M. B. STOUT, University of Michigan}

\author{
(Received November 17, 1937)
}

\section{SUMMARY}

Observations, taken during two winter storms with a group of sensitive anemometers, are presented and analyzed. Vertical sections of passing gusts for a height of $250 \mathrm{ft}$. and horizontal sections across a width of $660 \mathrm{ft}$. were obtained. Iso-velocity contours were drawn to show the structure of passing gusts. Contours were also drawn for theoretical cases of flow, from which it was possible to identify several aspects of vortical motion in the atmosphere.

$\mathbf{I}^{\mathrm{N}}$ NFORMATION of a new type regarding the structure of the wind has been obtained at the University of Michigan during the past several years. The investigations were undertaken in 1927 as part of a general research into the strength and the wind loading of electric power lines under storm conditions. ${ }^{1}$ The study was financed in the beginning by the National Electric Light Association, but since 1932 has been continued as independent research by the authors, with assistance from University research funds, the National Youth Administration, and the Airship Committee of the U. S. Navy. ${ }^{2}$

The material presented in this article was derived from two winter storms of considerable intensity. One of them, that of April 28, 1931, was analyzed only to the extent of showing wind structure diagrams for two gusts. The other storm, that of January 19, 1933, was more completely analyzed so as to include several wind structure diagrams; a study of the variation of wind velocity with height and the correlation with the gradient velocity; and a statistical study of gustiness.

\section{INSTRUMENTS AND METHODS}

The site of the experimental station is near Ann Arbor, Michigan, on a ridge of land whose axis is approximately normal to the prevailing storm winds. The windward side of the ridge has a gentle slope of about $175 \mathrm{ft}$. in 4 miles, and contains occasional farm buildings and groups of trees. The nearest obstruction is a grove at a distance of about $3 / 8$ of a mile, and at such an elevation that the tops of the trees are level with the top of the ridge.

A steel tower $250 \mathrm{ft}$. high was used with anemometers at 25 and $50 \mathrm{ft}$. intervals. Anemometers were also mounted on 12 poles $50 \mathrm{ft}$. high, spaced $60 \mathrm{ft}$. apart. From this arrangement it was possible to obtain a horizontal section of a passing gust at a height of $50 \mathrm{ft}$. above the ground and over a front of $660 \mathrm{ft}$. When some of the anemometers were placed on the tower in-

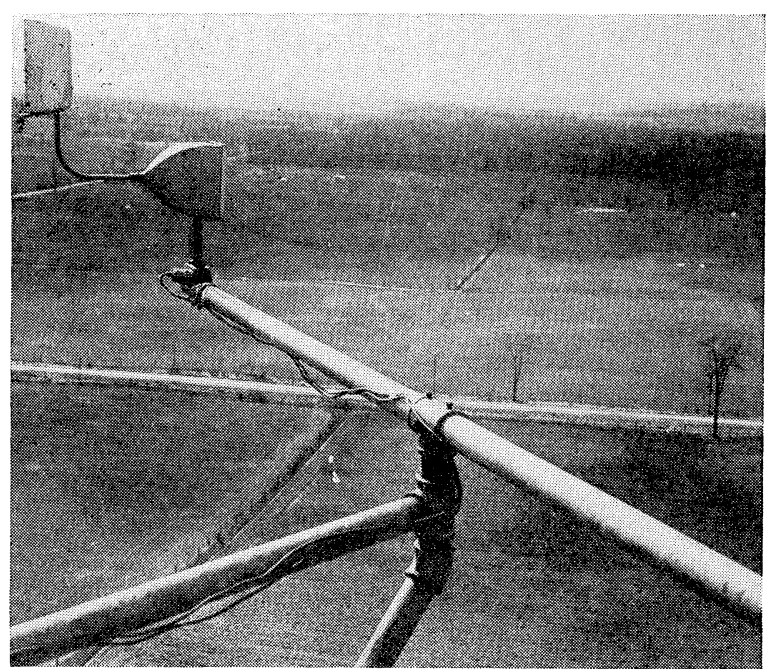

Fig. 1. Pressure plate anemometer installed on a tower station.

stead of on the wood poles, it was possible to obtain simultaneously a horizontal and a vertical section of the passing gusts.

\section{New Type Anemometers}

A study of the records of commercially available anemometers led to the conclusion that, because of the inertia of the moving parts of the instruments, the records could be accepted as accurate only if they were averaged over an interval of 10 seconds or more. This degree of responsiveness was not sufficient to disclose the characteristics of the passing gusts. An anemometer was therefore developed which would record accurately the average velocity for intervals of time as short as $1 / 4$ second or even shorter.

The new type of instrument has a pressure plate 9 in. high and 8 in. wide hinged near the bottom. Pressure against the plate is carried by a rod to a magnetic transmitter. The wind pressure changes the air gap of the transmitter, thus controlling the amount of current passing through its coil. The current was recorded by a 12-element oscillograph, which permitted the taking of simultaneous records from twelve anemometers.

The pressure plate has a maximum movement of about 0.005 in., and a natural frequency of vibration of about 115 cycles per second. About 0.12 second is required for the instrument to complete its response to a change of velocity from $80 \mathrm{~m} . \mathrm{p} . \mathrm{h}$. to zero. The extreme error due to temperature effects is 0.27 m.p.h.; that due 


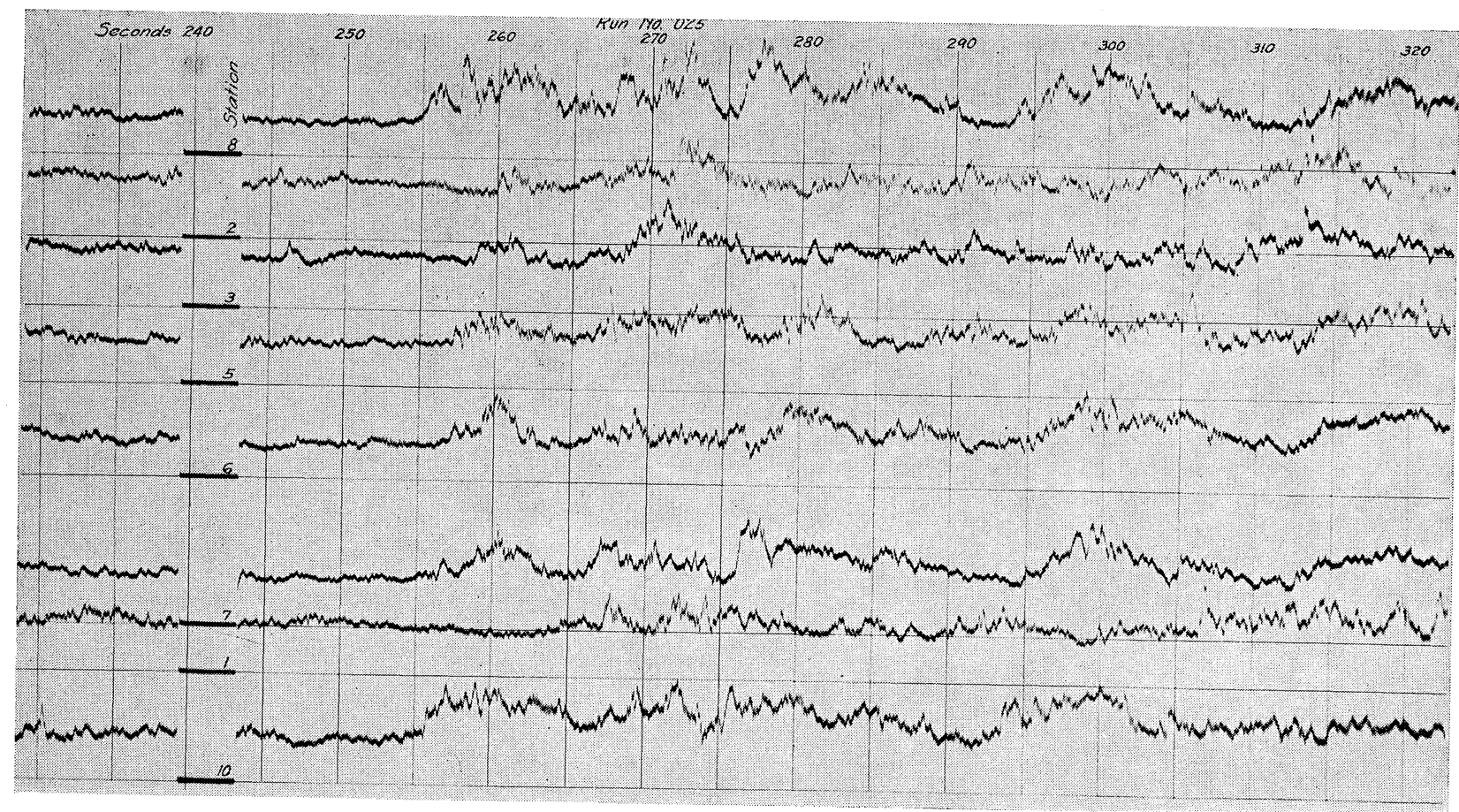

FIG. 2. Original chart, part of run 025 .

to imperfect leveling, 0.45 m.p.h.; and momentary errors due to the lag of the anemometer in responding to changes of wind direction, 1.25 m.p.h. A complete description of the anemometer and its recording instrument has already been published ${ }^{3}$ as well as a preliminary description of the method of drawing and interpreting wind structure diagrams. ${ }^{4}$ A photograph of one of the anemometers mounted on a tower station is shown in Fig. 1.

\section{Method of Taking Records}

The records from the 12-element oscillograph were made directly on sensitized paper which was obtained in rolls $12 \mathrm{in}$. wide and $100 \mathrm{ft}$. long. A chart speed of either 6 in. or 12 in. per minute was used, depending upon the intensity of the storm and the manner in which it was expected the records would be used. A typical record is shown in Fig. 2.

Transparent templates, inscribed with finely divided grids, were used to obtain data from the original charts. The zero-line of the grid was made to coincide with the electrical zero-line of the record and values of deflection were read through a magnifying glass. These values were entered in tabular forms and later converted to velocities through the calibration established for each combination of an anemometer with an oscillograph element.

The records of every storm were divided into runs of from 6 to 12 minutes each, with an interval of about one minute between runs. This article discusses wind gusts which occurred near the middle of Run 330 on the afternoon of April 28, 1931, and during Runs 025 and 026 in the storm of January 19, 1933.

\section{Diagrams of Wind Structure}

One of the most important objectives of this work was to obtain additional knowledge of the structure of wind gusts. For the purpose of showing the structure graphically, diagrams were prepared from the records in such a way that the character of the turbulence would be disclosed by inspection, as in Figs. 3 and 4. The data for these two diagrms are taken from Run 330, during the storm of April 28, 1931. Fig. 3 shows the variations that occurred in both a horizontal and a vertical section during an interval of 22 seconds. Spacing of the anemometers was used as ordinates, and time as abscissas. Time was divided into intervals of $1 / 2$ second and the average wind velocity in m.p.h. was recorded for each interval at each anemometer. Contour lines were drawn so that any given line represents a particular velocity and passes through only those periods in which that particular velocity was recorded by the anemometers. These lines, referred to as iso-velocity contours, indicate by their spacing the rapidity with which the velocity is changing.

A well-defined gust front appeared at Station 6 during the last half of Second 344 and spread to all the horizontal stations within the next 6 seconds. After the front had passed, the gust maintained a high average velocity at all stations with, however, rather rapid accelerations appearing in some places. It is interesting to note that the front of the gust reached the upper stations of the tower several seconds before it reached the $50 \mathrm{ft}$. level, and that a strikingly symmetrical eddy occurred near the top of the tower immediately following the front of the gust. These conditions are very similar 

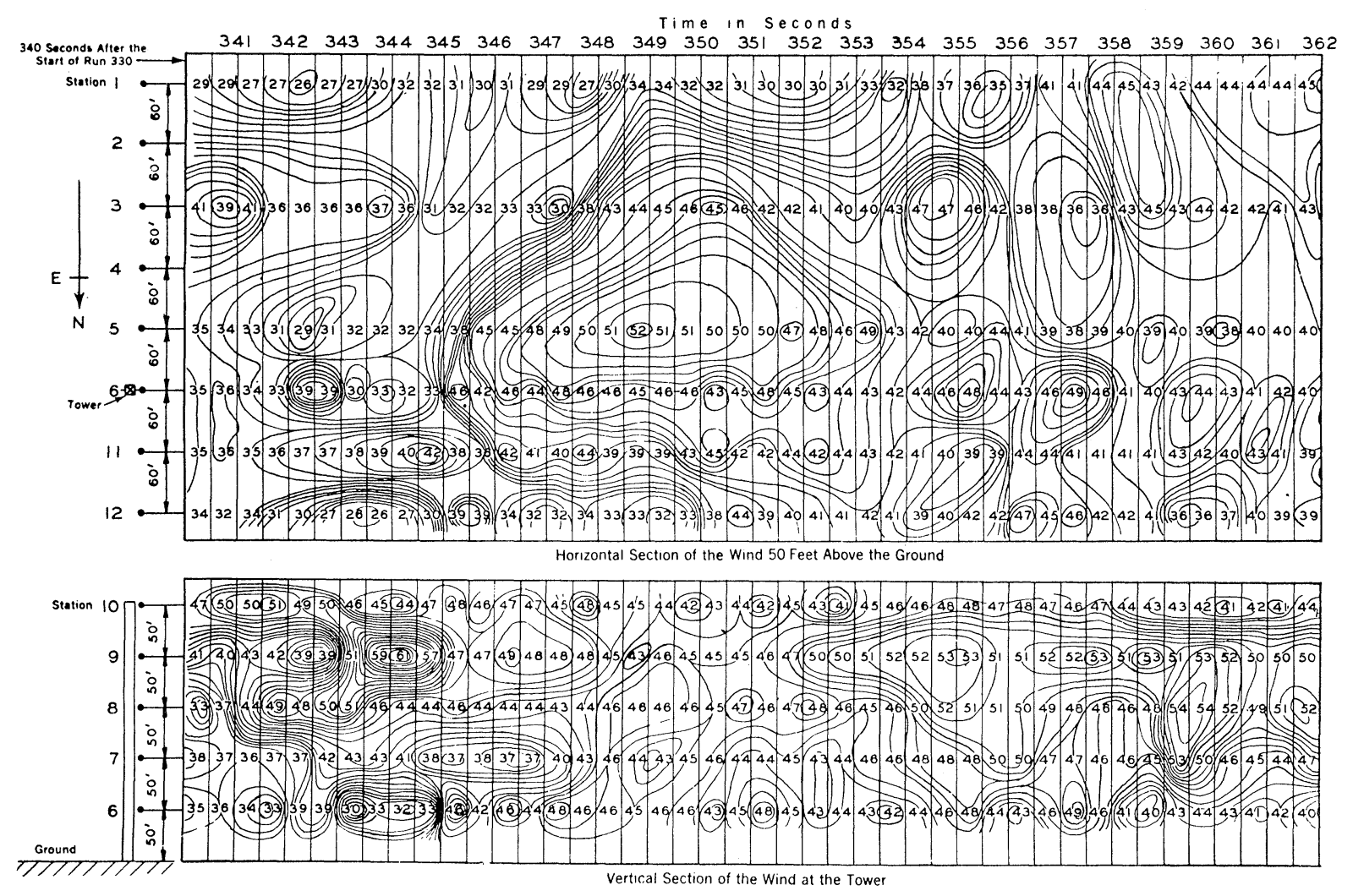

FIG. 3. Wind gust shown by iso-velocity contours, run 330. (Secs. 340-362.)

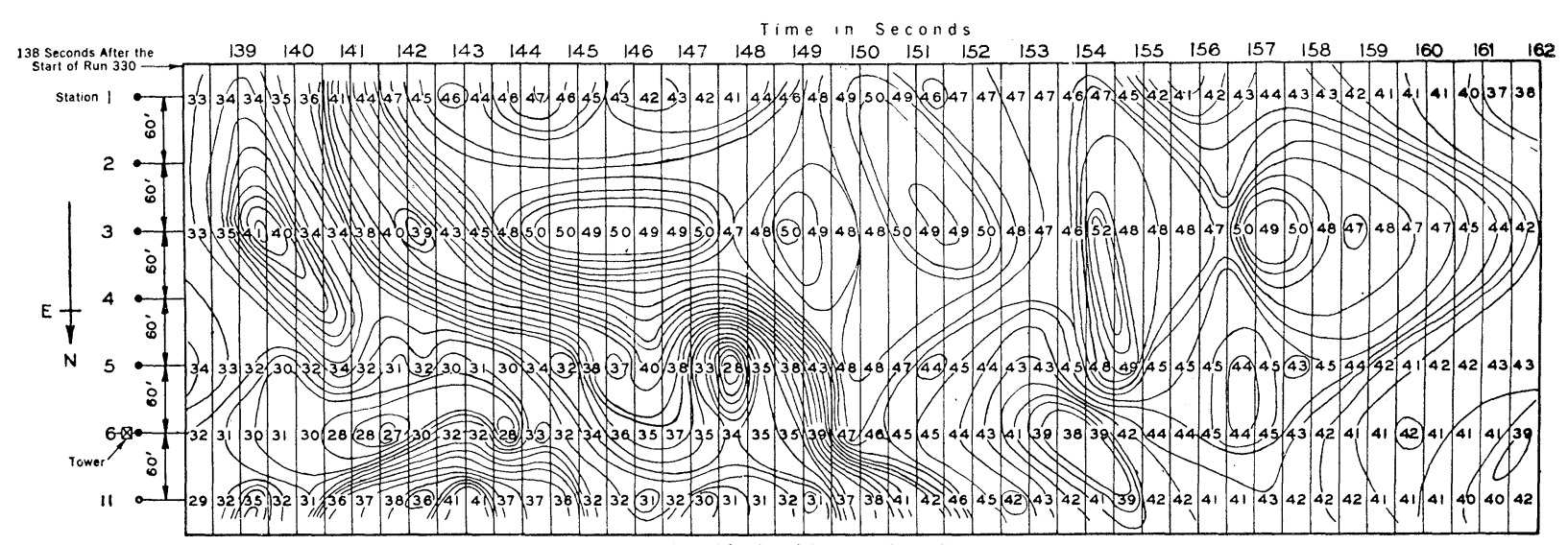

Horizontal Section of the Wind 50 Feet Abnve the Ground

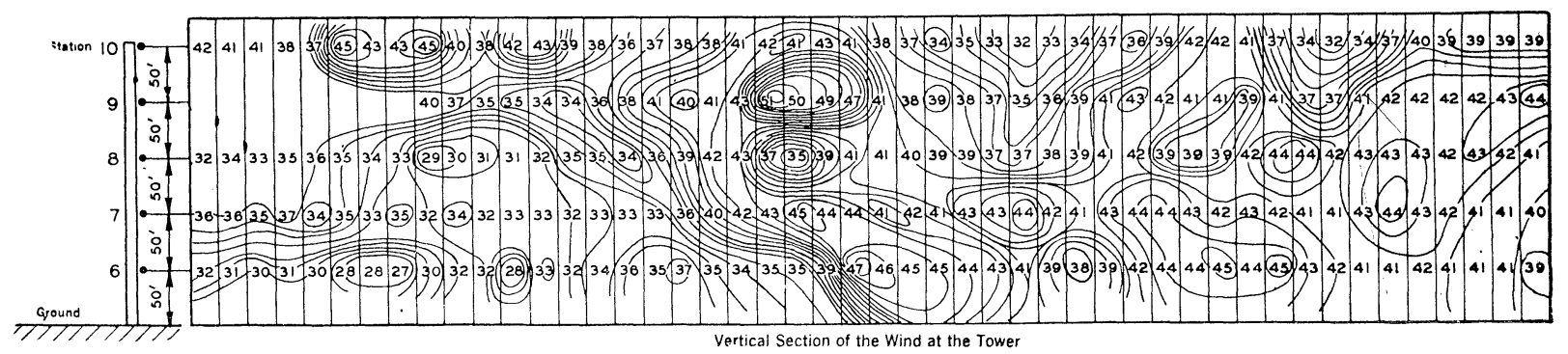

FIG. 4. Wind gust shown by iso-velocity contours, run 330. (Secs. 138-162.)

to those which existed at the beginning of an earlier gust, as shown in Fig. 4, except for the relative positions of the zones of high and low velocity. This combination of zones of high and low velocity occurs frequently throughout all the diagrams, particularly in the region immediately following the passage of the gust front. The relative position of the high and low velocity zones in the configurations varies considerably, but there is a similarity which suggests that they represent different aspects of a common type of flow. 


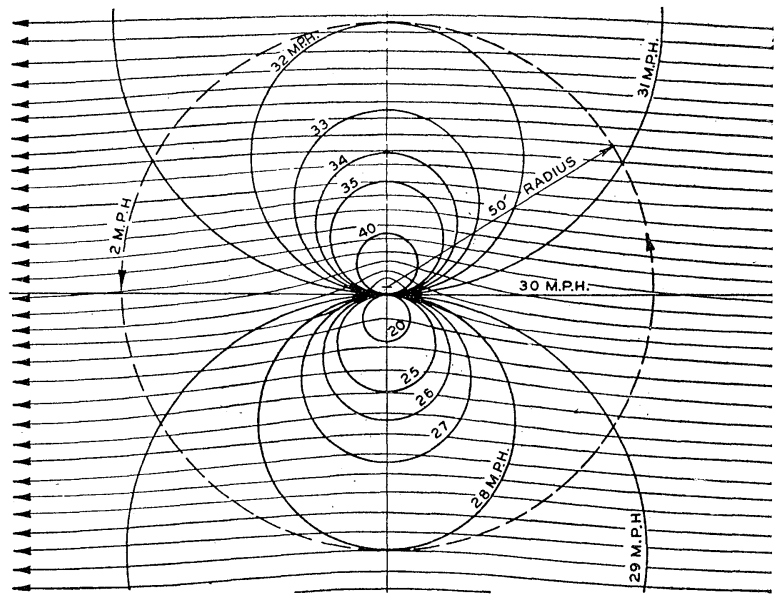

FIG. 5. Vortex superimposed on uniform flow.

\section{Theoretical Types of Eddies}

Iso-velocity contours for several theoretical cases have been constructed for the purpose of identifying the types of flow disclosed by the regular configurations in the wind diagrams. Several such cases will now be discussed.

In Fig. 5 are shown the stream lines and iso-velocity contours near a vortex whose center is traveling at the rate of 30 m.p.h. The strength of the vortex is such that at a distance of $50 \mathrm{ft}$. from its center the tangential velocity is $2 \mathrm{~m} . \mathrm{p} . \mathrm{h}$. The resultant velocity at any point is obtained by taking the vector sum of the two component velocities. There is a zone of high velocity in the upper half of the diagram and a zone of low velocity in the lower half, with a steep velocity gradient between them. Since one of the characteristics of a vortex is that the tangential velocities increase toward the center inversely as the radius, there is, theoretically, a point at which the tangential velocity is just equal to the velocity of translation, giving, in this case, a point of zero velocity a short distance below the vortex center. Between this point and the vortex center the resultant velocity is in the direction opposite to the velocity of translation.

As the velocity increases toward the center of the vortex there is a decrease in the static pressure, and consequently a necessity for air to rush into the open ends of the vortex cylinder and to form sinks. This involves a tendency for the vortex to disintegrate through the equalization of the pressure differences necessary to maintain it. In the natural air there is sufficient viscosity to slow down the disintegrating process and to permit the vortex to maintain itself by losing at its circumferential boundary an amount of air equal to that which flows into the ends of the cylinder.

Stream lines and iso-velocity contours for the theoretical case of an aerodynamic sink traveling at the rate of 30 m.p.h. are shown in Fig. 6 . The velocity of translation is parallel to the plane of reference and the velocities in the sink converge from all directions to the cen-

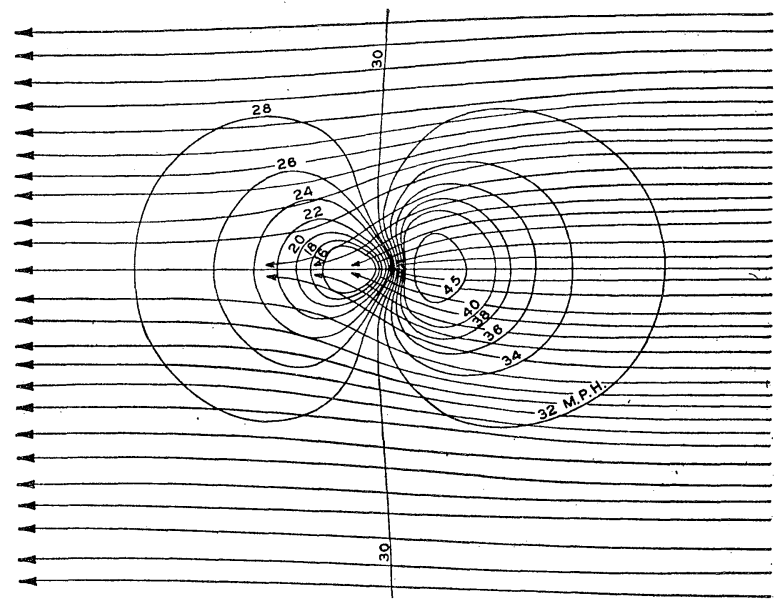

FIG. 6. Sink superimposed on uniform flow.

ter, which is $10 \mathrm{ft}$. away from the plane of reference. The strength of the sink is such that about $92,000 \mathrm{cu} . \mathrm{ft}$. of air per second disappear at its center from all directions. The stream lines and iso-velocity contours were obtained by taking the components of the velocities in the sink as they pass the plane of reference, and combining them vectorially with the velocity of translation in the plane.

Each of these two theoretical cases has a zone of high velocity and a zone of low velocity. In the case of the vortex, the two zones are side by side in the stream, but in the case of the sink the high zone follows the low zone. In both cases there is a steep velocity gradient between the two zones. In the case of the sink, the high zone tends to envelop the low zone, the amount of this enveloping tendency depending upon the relative strengths of the sink and of the uniform flow.

Two additional theoretical cases are pictured in Figs. 7 and 8. The combinations have been suggested by configurations of the iso-velocity contours appearing frequently in the wind structure diagrams. Fig. 7 is a combination of a vortex, having a counterclockwise sense of rotation, with a sink and uniform flow. Fig. 8 is a combination of a vortex, having a clockwise sense of rotation, with a source and uniform flow. In each case the vortex has the same strength as the one shown in Fig. 5, namely, 2 m.p.h. at a distance of $50 \mathrm{ft}$. from the center; the source and the sink each has the same strength as the sink in Fig. 6, namely, 92,000 cu.ft. of air per second, and in each case the velocity of translation is 30 m.p.h.

It will be seen that the chief difference between the configurations resulting from 3 components of flow and those resulting from 2 components is that the axes, joining the high- and low-velocity zones, occupy diagonal positions with respect to the direction of translation. In all cases the iso-velocity contour for 30 m.p.h., the velocity of translation, establishes the division between the high- and the low-velocity zones. This is important since it provides a means of identifying the velocity 


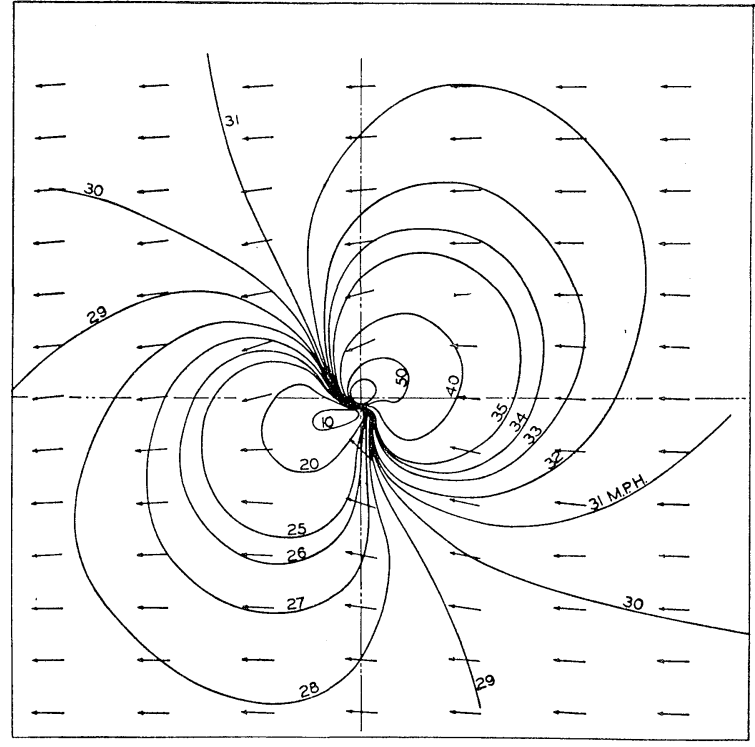

FIG. 7. Counter-clockwise vortex, sink and uniform flow.

of translation of the eddies appearing in the wind structure diagrams.

The arrows in these theoretical fields indicate the direction of flow. It is important to notice that large deviations from the direction of translation appear only near the center of the vortex or sink, or any combination of vortex, source, sink, or translation. In other words, even if the eddies in the atmosphere were visible to the eye, it would be possible to identify only a very small core of the eddies since, at all other points in the field, the direction of flow is substantially that of the direction of translation. Of course it would be possible to increase the size of the visible core in the diagram by increasing the strength of the vortex and decreasing the velocity of translation. However, the velocity of translation and the strength of the vortex, source, and sink were chosen so as to produce iso-velocity contours comparable to those disclosed by the records.

\section{Eddies in the Atmosphere}

The cases of the vortex and the sink are easily observed in water because the direction and velocity of flow are visible. In the case of water flowing into a vertical drain pipe, as in a kitchen sink, the characteristics of both a vortex and a sink are present. In the natural wind, however, these phenomena are not visible, except when the air is laden with snow or dust, at which times the core of the vortex is frequently outlined quite sharply, showing not only the tangential velocities of the vortex, but also the inflow or sink at its bottom boundary, and the outward flow, or source, higher up where disintegration is apparently taking place.

It is reasonable to expect that the theoretical cases will rarely be reproduced perfectly in the natural wind, since eddies in the wind stream are constantly undergoing changes from various causes. Furthermore, this

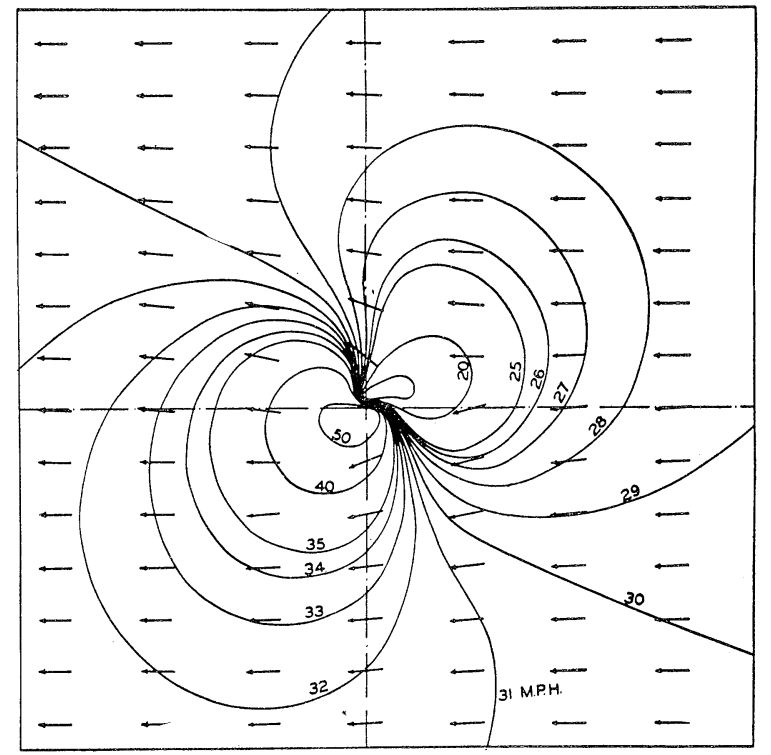

FIG. 8. Clockwise vortex, source and uniform flow.

method of measuring and recording the structure of wind gusts shows the shape of the eddies as they pass the line of anemometers and not as they exist in their entirety in any instant. If, therefore, an eddy starts to disintegrate or to combine with another eddy just as its boundary reaches the anemometers, the configuration of the iso-velocity contours in the upstream part of the eddy will be less perfect than in the downstream part. Moreover, a vortex may present many apsects besides the four theoretical cases treated here. Its flow may be two dimensional or three dimensional; its axis may be straight, curved, horizontal, vertical, or inclined.

\section{Storm of April 28, 1931}

By reference to Fig. 4, Secs. 147 to 150, it will be seen that the configuration of the iso-velocity lines at the upper stations of the tower indicates the presence of a vortex whose axis is approximately horizontal and whose direction or rotation is counterclockwise. There is a well-defined zone of high velocity and one of low velocity with a gradient amounting to $16 \mathrm{~m} . \mathrm{p} . \mathrm{h}$. in a vertical distance of $50 \mathrm{ft}$. This vortex is generated chiefly by the difference in the velocities of the upper and lower strata, as the lower strata lagged behind the upper because of friction with the ground and the viscosity of the air.

A well-defined sink, which appeared at the upper stations of the tower during Secs. 341 to 345 is shown in Fig. 3. Here the zone of high velocity follows that of low velocity in the wind stream, and a very steep velocity gradient exists between the two zones. The tendency for the high zone to envelop the low zone is more pronounced here than in the theoretical case shown in Fig. 6.

There are several places in the horizontal sections of the gusts in Figs. 3 and 4 where the configurations are 


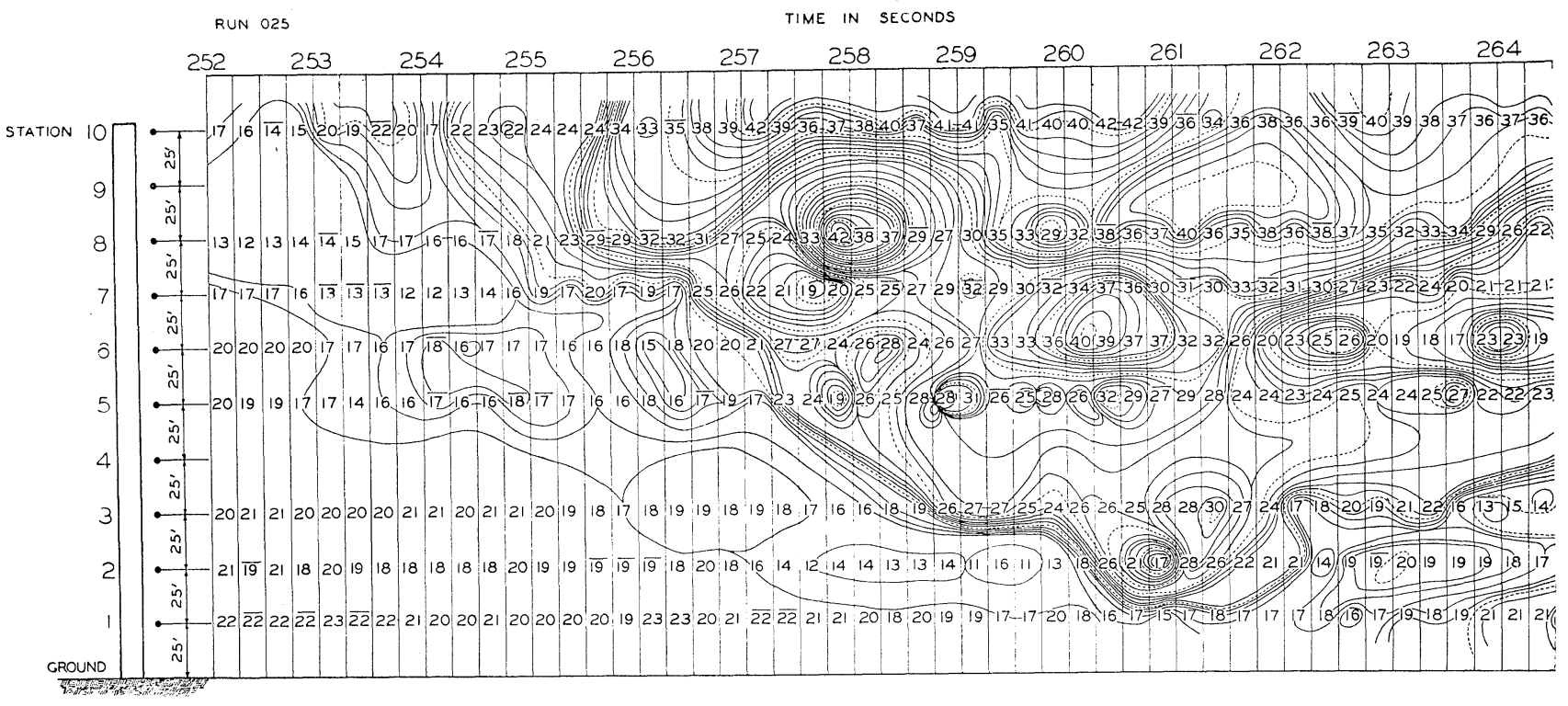

FIG. 9. Vertical section of

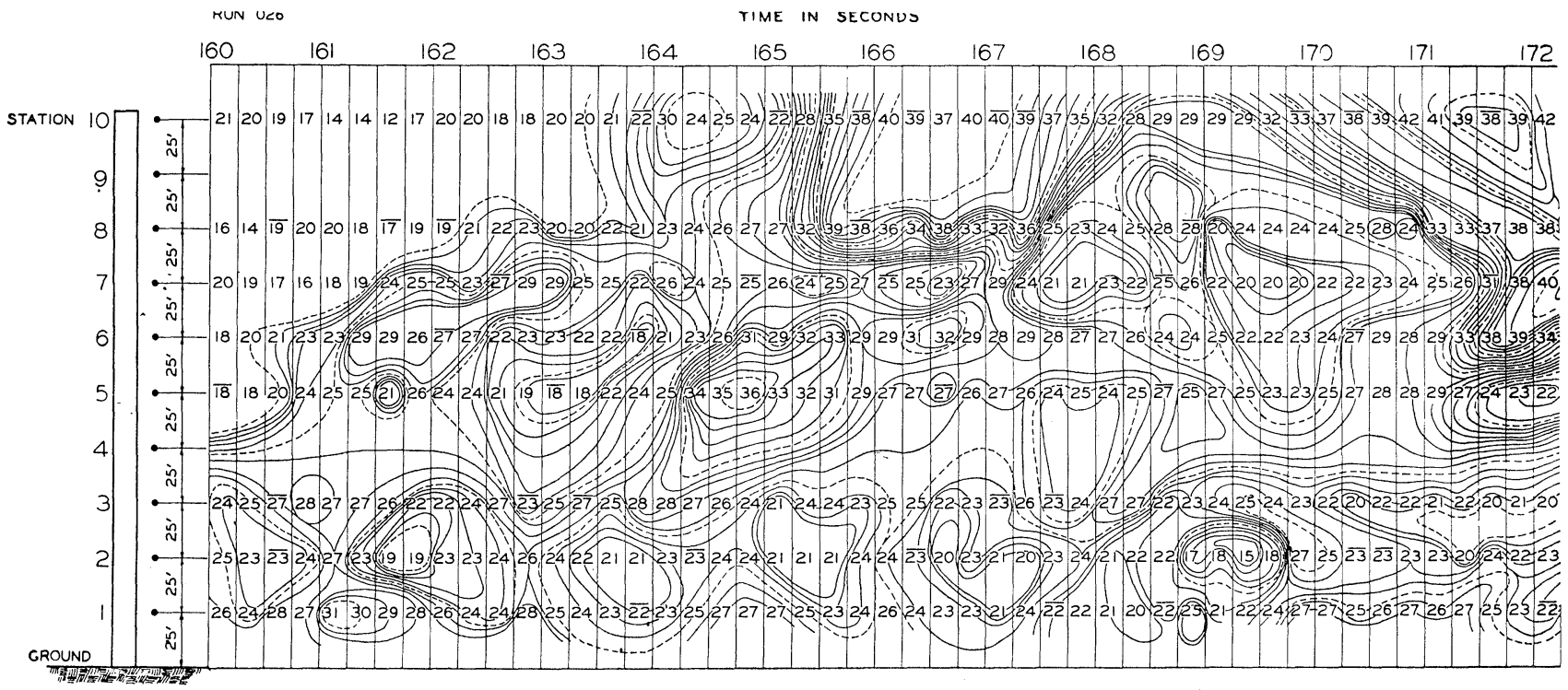

FIG. 10. Vertical section of

similar to the theoretical configuration of a vortex combined with a sink and uniform flow, as, for example, during Secs. 152 to 156 and 356 to 360 . Such a combination of flow may very well exist in the case of a vortex whose axis is nearly vertical, and whose lower boundary is near the plane of reference. Under this condition the horizontal section near the bottom end of the vertical vortex cylinder would include the upward velocity components of the inflowing air as well as the tangential components of the vortex.

There is an upper and a lower limit to the size of the eddies which can be disclosed by any given combination of anemometer spacing and length of time interval. For example, the combination of 50 and $60 \mathrm{ft}$. spacing of the anemometers with time intervals of $1 / 2$ sec. is about the least refined combination which could have been used without losing all indication of the presence of the eddies which are here disclosed. If the spacing of the anemometers on the tower had been $100 \mathrm{ft}$. instead of $50 \mathrm{ft}$. the vortex during Secs. 147 to 150 would have appeared merely as an irregularity in the iso-velocity contours.

\section{Storm of January 19, 1933}

After the winter of 1930-31, the chart speed was increased in order that time intervals of $1 / 4 \mathrm{sec}$. might be used on any records that involved high velocities. The eddies which are barely disclosed in the records in Figs. 3 and 4, with average velocities between 35 and 40 m.p.h., would be completely lost in records with higher average velocities, unless the length of time intervals were decreased as the velocity increases. Furthermore, the full benefit of decreasing the length of the time intervals would not be obtained without a corresponding 


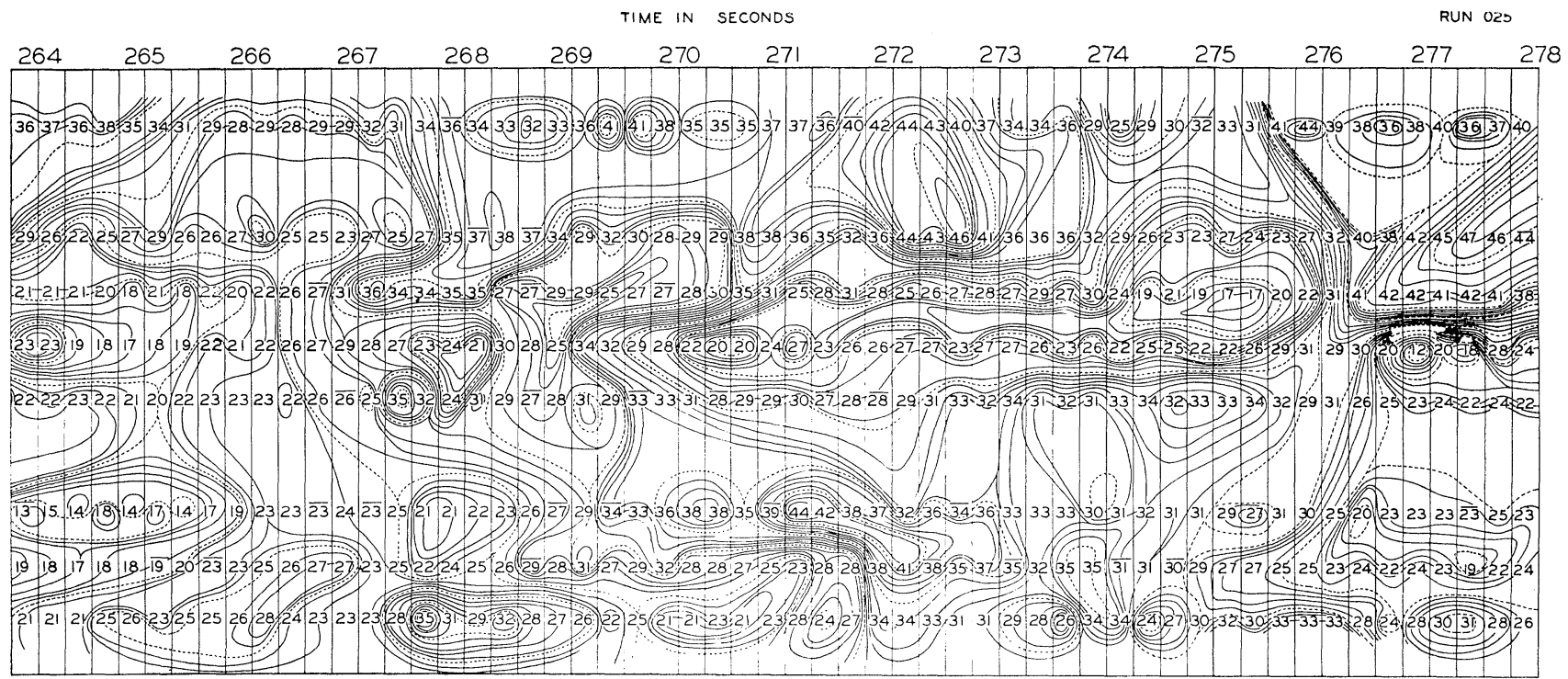

gust, run 025. (Secs. 252-278.)

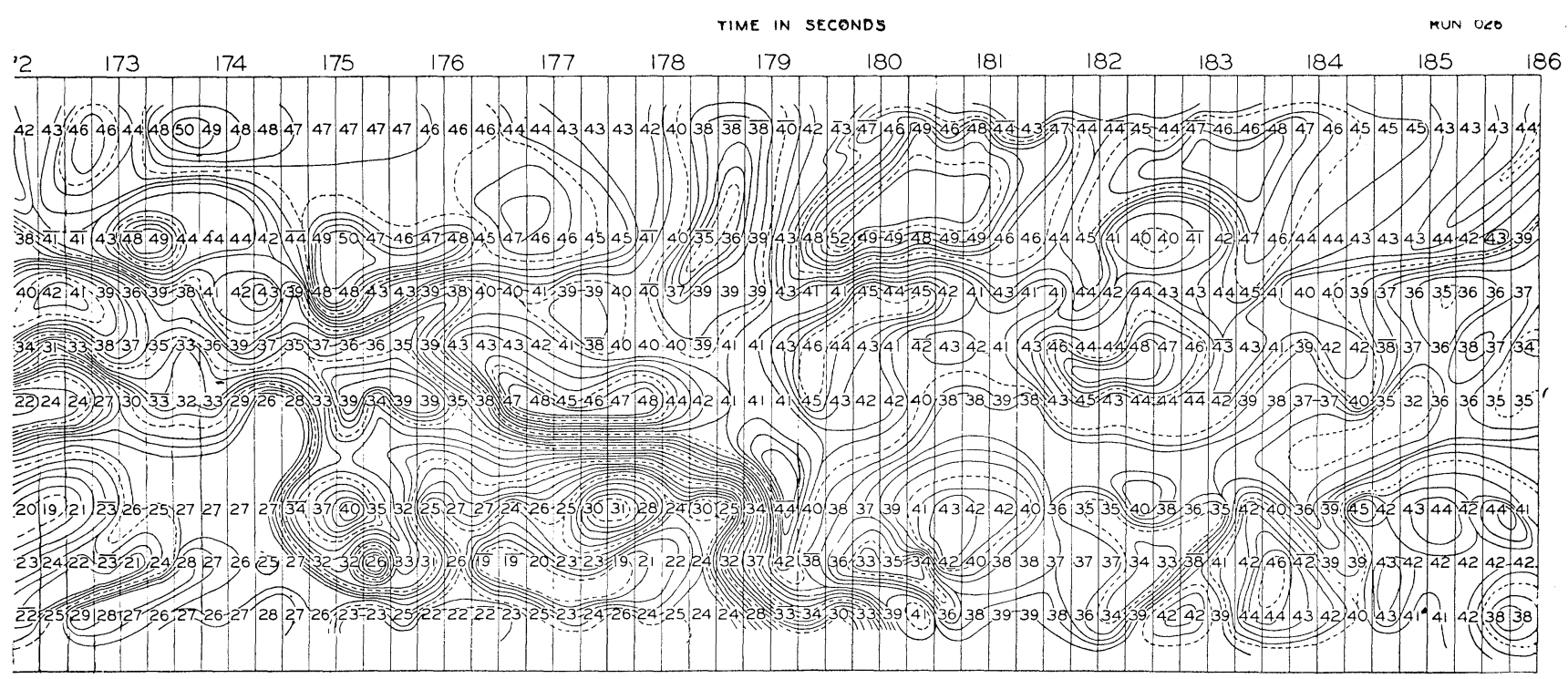

gust, run 026. (Secs. 160-186.)

decrease in the spacing of the anemometers. Consequently, during the storm of January 19, 1933, the spacing of the anemometers on the tower was $25 \mathrm{ft}$., thus giving 10 stations instead of 5 .

Figs. 9 and 10 are wind diagrams for the storm of January 19, 1933. It will be noticed that the numbering of the stations on the tower has been changed from that of the storm previously described; also, that the records of wind velocity are missing for the stations at the 100 and $225 \mathrm{ft}$. levels. Each number in the diagram represents the average velocity for a one-quarter second interval of time, instead of one-half second as previously used.

Fig. 9, Secs. 252 to 278 , shows the front of a gust and a small part of the structure following the front. Preceding the gust there is a period of about 40 seconds during which the wind velocity was relatively low and free from turbulence. This is clearly shown on the original record in Fig. 2. The highest average velocity within this inactive period was at the lowest station, no doubt because of the upward deflection of the air by the sloping ground in front of the tower. The first onslaught of the gust did not reach the lowest station on the tower, but receded after about 10 seconds to a height above the $250 \mathrm{ft}$. level. After a short interval a second onslaught of the gust succeeded in pentrating to the lowest station. More than 12 seconds elapsed after the gust had reached the highest station before it was fully evident at the lowest.

Following the gust front, there is a well-defined combination of vortex and sink at Stations 7 and 8 . The velocity of translation of this eddy was about $27 \mathrm{~m}$.p.h., as shown by the iso-velocity contour separating the highvelocity zone from the low one. It is impractical to com- 
pare the velocity of translation of a vortex with the average of the stream because of the difficulty of choosing a significant time interval over which to take the average of these turbulent velocities. The behavior of vortices seems to depend more upon rapid changes in velocity, and upon steep velocity gradients between stations, than upon anything which can be characterized by an average velocity.

Other configurations are present in the wake of the gust front, but they possess less regularity than the first one. For example, configurations indicating the presence of a vortex and sink are shown at Stations 5 and 6 , Sec. 258 , and a counterclockwise vortex in combination with a source during Secs. 267 and 268.

The presence of eddies can be detected in some cases on the original record, as in Fig. 2. As an example, the original record for Station 8, Secs. 257 and 258, shows quite distinctly the characteristic drop as the zone of low velocity passes the station, followed by a very steep velocity gradient as the front of the high velocity zone passes, and this in turn followed by the decreasing velocity as this latter zone recedes. The dip in the curve as the low velocity zone passes Station 7 is less jagged in appearance because the center of the vortex is not as close to it.

Fig. 10 shows a vertical section for Secs. 160 to 186 , Run 026. Here again the front of the gust made several onslaughts before it succeeded in penetrating to the lowest station. The gust front has the appearance of irregular masses of air tumbling down from above. The velocities are, in general, higher than those shown in Fig. 9 .

\section{Discussion of Wind Diagrams}

An inspection of the diagrams, and a comparison with the diagrams of theoretical cases, leaves no doubt that eddies of many different sizes exist in the turbulent atmosphere. The conditions which produce eddies and control their size have not yet been determined experimentally. The identification of these conditions awaits the more intensive study of existing records and the accumulation of additional records covering different size ranges. Indefinitely smaller eddies could be discovered by using closer spacing and smaller time intervals, but, as stated before, care must be used to preserve a proper relation between the spacing, time interval, wind velocity, and size of the space to be explored.

It is not certain whether the eddies are generated chiefly by steep gradients in the horizontal components of wind velocity or whether the vertical components also have an important influence. It is probable that the latter statement is correct because vertical components of velocity must exist as the front of the gust cuts its way downward toward the ground. However, the records on this investigation contain no information regarding vertical velocities since the pressure-plate anemometer rotates about its vertical axis only. It records the true resultant velocity only when the wind direction is horizontal. It is recognized that an error is introduced into the recorded horizontal component by any inclination of the resultant away from a normal to the pressure plate. The limiting magnitude of such errors has been determined experimentally for angularity in a horizontal plane, but not in a vertical plane.

In order for the surge to have reached the $50 \mathrm{ft}$. station within 5 seconds after it had reached the $200 \mathrm{ft}$. station, as shown at the front of the gust in Fig. 9, the effect must have traveled downward with a vertical velocity of about $30 \mathrm{ft}$. per sec., about $20 \mathrm{~m}$. p.h. This effect could be produced through shear between horizontal layers, or through vertical displacement of masses of air having different velocities, or through a combination of these two means.

The phenomenon of vertical interchange due to thermal instability is well known. Violent gusts of thermodynamic origin, involving the vertical movement of large masses of air, occur during the passage of the areas of low barometric pressure. During the storms recorded in this investigation the highest velocities and the most violent gusts occurred within the southwest quadrant of low-pressure area, that is, while the cold front was passing, and during the time when the low-pressure area was receding and the high-pressure area was approaching. In this zone of transition there was a condition of thermal instability which permitted occasional and sometimes violent interchanges between the higher and lower strata. Large gusts near the ground occurred because masses of air from the more rapidly moving higher strata reached the ground before losing all of their excess forward momentum.

Experimental studies have been made by others to obtain quantitative information about the structure of the wind. All of these attempts have been limited in their scope and accuracy by the instruments available to the investigators. One of the most recent and most successful investigations of this kind was conducted by Wilhelm Schmidt. ${ }^{5}$ By taking motion pictures of a line of pendulum plates and observing the angles through which they were deflected, he was able to determine the average wind velocities existing at the various plates for intervals of time as small as $1 / 7$ of a second, if the wind velocity did not exceed 10 meters per second (22.4 m.p.h.). The length and height of the front over which observations can be made in this way are rather limited. The purpose of his investigation was served by taking simultaneous records for a height of 1.5 meters and a width of 3 meters in one case, and for a height and width of 10 meters in another case.

For the purpose of showing graphically the structure of the wind Schmidt likewise used spacing of the anemometers as the ordinates and time as the abscissas. He indicated the zones of different velocity by different types of cross hatching. In his diagrams there were no indications of the presence of vortices. From the published results it is not clear whether this is due primarily to (1) the fact that his diagrams did not include gust fronts, 
involving large differences in velocities; or (2) sluggishness of the pendulum plates; or (3) the fact that the time intervals over which his velocities were averaged were too large to disclose any eddies which could be encompassed in the relatively small dimensions of his apparatus. The last of these would seem to be sufficient explanation. The height of his time-space diagrams was 1.5 meters. When the velocity was 10 meters per second a vortex with a diameter of 1.5 meters would pass his pressure plates in a period of 0.15 seconds. At the rate of 7 readings per second only one reading would be obtained during the passage of a vortex. Even for velocities of translation as low as 3 meters per second, only about three readings would be obtained during the passage of the eddy, and these would scarcely be sufficient to identify it.

The arrangement and responsiveness of the anemometers on the investigation described in this article, permitted not only the mapping of wind structure, but also the identification of several aspects of vortical motion. It has been possible to identify a number of cases in which the regularity and shape of the iso-velocity contours approach that of the theoretical cases. The wind diagrams may also be used for purposes other than the identification of particular forms of air flow. The presentation of wind data in this form shows both the varia- tion of velocity with time at a given place, and the variation in space at any instant of time. Wind pressure may be evaluated from the velocity data, yielding information as to the dynamic characteristics of gust forces, and the width or height affected by them. The original tabular collection of data, however, opens an even wider field of investigation which is still being explored, namely, the computation of correlation coefficients and other statistical quantities which may be used to search for orderly relationships in the apparently disordered atmosphere.

\section{REFERENCES}

${ }^{1}$ Sherlock, R. H., and Stout, M. B., Storm Loading and Strength of Wood Pole Lines, Edison Electric Institute, 1936.

${ }^{2}$ A more detailed report has already been made to the Airship Committee, Prof. W. F. Durand, Chairman, and may be published later by the committee. The parts dealing with the variation of velocity with height and statistical analysis of turbulence have been omitted from this article, but they are being included in Engineering Research Bulletin No. 28, University of Michigan now being prepared for publication.

${ }^{3}$ Sherlock, R. H., and Stout, M. B., An Anemometer for a Study of Wind Gusts, Engineering Research Bulletin No. 20, University of Michigan, May, 1931.

${ }^{4}$ Sherlock, R. H., and Stout, M. B., Picturing the Structure of the Wind, Civil Engineering, June, 1932.

${ }^{5}$ Schmidt, Wilhelm, Turbulence Near the Ground, Jour. Rov. Aero. Soc.. May, 1935. 